\title{
Rainer Erd Auf dem Weg zu einem europäischen Kulturverwaltungsrecht
}

Daß Kultur verrechticht werden kann, will nicht unmittelbar einleuchten. Denn im Gegensacz zu den klassischen Gegenscänden von Verrechulichung, dem Privareigenrum, dem Warenverkehr und dem Verhältnis des Bürgers zum Staat, enchält Kultur etwas Spezifisches. Als Ausdruck eigensinnigen, sich den warenjörmigen Beziehungen von Menschen cntzichenden Handelns, scheint Kultur nicht verrechtliclibar zu sein. Ist für die recbtsförmige Verarbeitung individueller oder sozialer Probleme ein gewisses Maß an Kalkulierbarkeit Voraussetzung, soll will Kultur im empharischen Sinne gerade das Gegenceil, das Unkalkulierbare scin. "Kultur«, schreiben Adorno und Horkheimer, als das über das System der Selbsterhaltung der Gatcung Hinausweisende, enthält allem Bestchenden, allen Insticutionen gegcnüber unabdingbar ein kritisches Moment."' Andererseits freilich kann Kultur ohne Formen der Insticucionalisierung daucrhafo nicht exiscieren. Wenngleich die Warenbezichungen von Menschen überschreitend, ist Kultur doch auch efwas Kontinuierliches, Kalkulierbares. Denn ahne kulturelle Ausdrucksformen, in denen sich Probleme und Sehnsüchte von Menschen reflektieren, ist soziales Leben nicht möglich. Im Gegensatz zum Warenverkelır freilich, dessen Regeln mit rechclichen Sankiionen geltend gemacht werden können, sind kuluurelle Normen allenfalls moralisch reklamierbar. Eine nicht erbrachte Leiscung kann gerichclich eingeklagt wcrden, ein Verstoß gegen kulturelle Normen ist allein sozial sanktionierbar.

Als etwas Kontinuierliches, aber nicht Kalkulierbares scheint sich Kultur eincr Verrechtlichung gegenüber zu sperren. Dennoch ist eine umfangreiche Praxis von Kulturvervaleung unübersehbar. Wie bürokratisch verzerr Kultur in verwaltungsförmiges Handeln gepresst werden mag, daß es Kulturverwaltungen gibt, läßt sich nicht leugnen. Und diese sínd Kultur nicht allein aufgezwungen, sondem garantieren vielfach auch mit Zustimmung der Betroffenen, daß Kultur überhaupt bestehen kann. "Wer Kulur sage«, so Adorno und Horkheimer, "sagt auch Verwalcung. «" Denn ohne staathche Garantie droht Kultur ihre Existenz im Kommerz zu verlicren oder von privazen Mäzenen abhängig zu werden. Eine Beschäfrigung mit Problemen der Verrechtlichung von Kultur muß deshalb von dem paradoxen Sachverhalt ausgehen, daß Kultur, die ohne Autonomie nicht gedeihon kann, sich auf der cincn Seite gegen Verrechtlichung sperrt, auf der anderen Seite ohne recheliche Normicrung schwerlich existenzfähig ist. Die Ambivalenz von notwendiger, aber begrenzter Verrechtlichung wird dic Skizze der Probleme cines europäischen Kulturverwaltungsreclits leiten. Kulturverwaltungsrecht bezieht sich nicht auf die Inhalte von Kultur, sondern regelt die institutionellen Bedingungen ihrer Autonomic. Dies kann in Form materieller Zuwendungen oder der Sicherung vor staatichen und gesell-

1 Kulcur und Venaltung. in: dies.. Sociologica II. Reden und Varträge, Frankfurı am Marn 1962, 5. s5. 2 A. A. O., $\$ .48$. 
schaftlichen Einschränkungen geschehen. Kulturverwaltungsrecht ist vorwiegend Subventions- und Schutzrecht.

Ausdruck der Ambivalenz des Kulturverwaltungsrechts ist die offensichdich ungleiche Bewertung kultureller Problene in der recheswissenschafelichen und der sozialwissenschaftlichen Diskussion. In den Sozialwissenschaften bat in den vergangenen Jahren ein Wechsel der Problemfelder stattgefunden. Ließen sich in den sechziger und siebziger Jahren zentrale Probleme sozialwissenschafclicher Theoriediskussion um das Begriffspaar "Wirrschaft und Kulcura gruppieren, so ist an dessen Seclle zunehmend das Problemfeld "Kultur und Staat "gerreten. Früher weithin verwendece Katcgorien wie Mehrwerr, Ausbeutung, Klasse und statliche Repression entstammen dem theorerischen Umfeld von "Wirtschaft und Staat*. Heute in den Sozialwissenschaften übliche Kategorien wic Individualisierung, Autonomie, Lebensstil und politisches System leiten sich aus theorecischen Überlegungen her, die in der Diffusion traditioneller Klassenstrukruren und der Herausbildung teilautonomer, über kulturelle Erfahrungen vermitceler sozialer Bereiche das Spezifikum gegenwäriger Geselischaften sehen. In den Rechtswissenschaften hat der sozialwissenschaffliche Kategorienwechsel kein Pendant gefunden.

Wer sich auf die Suche nach umfassenden Veröffentlichungen aus dem Bereich der Verrechelichung von Kultur macht, wird allein auf zwei Publikationen stoßen, von denen dic für unseren Zusammenhang bedeutungsvollste $20 \mathrm{Jahre} \mathrm{alt} \mathrm{ist,} \mathrm{ohne} \mathrm{bisher}$ einc Neuauflage erfahren zu haben.' Die Tatsache, daß Oppermanns Lehrbuch zum Bildungs-, Wissenschafts- und Kunstrecht nunmehr zwei Jahrzehnte unverändert überstanden hat und daß auch die dort angekündigren jährlichen Ergänzungen in der ojurisrenzeirung* niemals erschienen sind, kann als Ausdruck der für die Kultur charaktcristischen Ambivalenz von Autonomie und Verrechtlichung verstanden werden. Diese vergrößerr sich, wenn von Kultur auf europäischer Ebene die Rede ist. Denn nun treten neben die prinzipielle Autonomie von Kultur je spezifische nationale Besonderheiten, die cincr Verrechtlichung weitere Hindernisse in den Weg lcgen.

\section{Probleme eines europäischen Medienrechts}

Die skizzierte Ambivalenz einer Verrechtlichung von Kultur auf curopäischer Ebene soll am Beispiel des Medienrechıs näher aufgezeigs werden. Im Zentrum der Diskussion steht das Problem der Quottering von Rundfunk- und Fernselisendungen. Aul den ersten Blick widerspricht der dem Quorierungsmodell zugrundeliegende Gedanke der Idee einer die nationalen Grenzen überschreitenden Kooperntion. Quotierung im Medienbercich bedeutet, daß der nationale Gcserzgeber einc bestimmte Quote festserzt, die zu überschreiten ausländischen Kulturproduzenten untersagt ist. Wälrend das Konzept eines kulturellen Europas gerade auf der Bescitigung nationaler Schranken und der Erleichterung des Austauschs von Kulturgürern basier, will das Quotierungsmodell spezifische rechtliche Restriktionen cinführen, die den ungehinderten Kulcurausıausch einschränken sollen. Europäisierung der Kultur und Quotierung scheinen im Widerspruch zueinander zu stehen. Die Befürworter von Quoticrungen im Bereich der Medien sehen das freilich nicht so. Sie verwcisen auf die Notwendigkeir von Quoticrungen mit dem Argument, obne diese bestehic die Gelahr, daß bestimmte auswärtige Programme die nationale

3 Th. Oppermann, Kulturvenwaltungsritht. Bildung-Wissenschaft.Kunst, Tübingen 1969. 
Produktion zu dominicren drohen und so zu ökonomischen und kulturellen

Problemen führen. Konkret: Lege der Gesetzgeber nicht bestimmte Quoten etwa für amerikanische Fornsehscricn in deutschen Programmen fest, dann bestehe dic Gefahr, daß solche „Billigprodukrionen « das kulturellc Nivenu des Mediums Fernsehen senken und die deutsche Film- und Fernsehproduktion ökonomisch EinbuBen crleiden könne.

Wiervohl es in den siebziger Jahren in der Bundesrepublik bereits Diskussionen über Quoticrungen (besonders gegenüber Film-Synchronisacionsfirmen aus der DDR) gab, begann cine umfangreiche Diskussion darüber erst Anfang der achiziger Jahre. Hintergrund dieser Entwicklung war die in dieser Zeir einsetzende Diskussion über die Privarisierung öffentlich-rechtlicher Medien. Eine 1983 von der EG-Kommission vorgelegte Studic behaupter, daß unter der Annahme von zukünftig 36 Fernseh-, Kabel-und Satellirenkanälen in den Ländern der Europäischen Gemeinschaft jäbrlich ca. I 500000 Sendescunden produziert werden und vcrfügbar sein müßten. Der Bedarf nach neven Filmen und nach Fernsehuncerhaltung wurde auf jährlich 125000 Stunden geschärzt. Da dic wichugsten Produktionsländer der EG zusammen demgegenüber nur 5000 Stunden herstellten, vergrößere sich der Einfluß amerikanischer Produzenten dann weiter, wenn dieser nicht durch restriktive Regelungen cingeschränkt werde." Eine der Reaktionen auf diese Entwicklung war die verstärkte staatliche Filmförderung, häufig in Form einer intensivierten Kooperation von Filmproduzenten mit dem Fernschen. Die Quocierung stellte eine andere Antwort auf das Problem dar. Engagieric Befürworter einer Quocierung in öffentlich-rechtichen Medien sind keineswegs, wie man meinen könnte, nacionalistisch orientierte Kulcurpolitiker, sondern solche, die sich (wic Petcr Glorz und Alexander Klugc) der unorhodoxen Linken zugehörig fühlen.

Es dauerte jedoch noch knapp drei Jahre, bis das crstc deutsche Rundfunkgesetz (in Nordrhein-Wescfalen) mit einer Quotierungsregelung vom Geserıgeber verabschiedet wurde. In $\$ 12$ Abs. 5 des Gesetzes heißt es: "Jedes Fernsehvollprogramm soll zu einem überwiegenden Anteil aus Eigen- und Auftragsproduktionen, auch in Form von Gemeinschaftsproduktionen, bestehen. Es soll zugleich einen überwiegenden Anteil von Produktionen aus dem deutschsprachigen und europäischen Raum enthalten. Das Nähere kann die Landesanstalt für Rundfunk durch Satzung bestimmen; dabei ist vorzusehen, daß die vorhergeschenen Antcile stufenweise innerhalb mehrcrer Jahrc nach der Zulassung erreicht werden können. Die Veranstaltcr haben der LRR jährlich die in den cinzelnen Programmen errcichten Anteile anzuzeigen." Daß dic Befürchtungen des Gcsetzgebers nicht unberechrigt sind, läße sich einer Untersuchung aus dem Frühjahr 1986 entnehmen. Danach betrug bei den privaten Fernsehsendern unter den "Serien* der Anteil amerikanischer Produkrionen 100 (RTL plus) bzw. 83,9 (SAT 1), während er bei der ARD nur 27, 1 und beim ZDF 23,8 Prozent ausmachte. Auch bei den Spielfilmen war der Anteil ausländischer Produktionen um ein Vielfaches höher als bei den öffentlich-rechclichen Anstalten."

Was der nordrhein-westfälische Geserzgeber fixiers hat, ist - vergleicht man es mit anderen westcuropäischen Ländern - keine ungewöhnliche Regelung. Die Reglementierung des Auslandsanteils in Hörfunk und Jernsehen hat erwa in Frankreich einc lange Tradition. Dort legen Mediengesecze Rahmenbedingungen für Rundfunkprogramme fest, bestimmt dic Regicrung Quoten für spezifische Programmfor-

4 Zituer nach: A.J. Wiesand, Kunst ohne Grenxen? Kulturelle ldenutả und Frezzügigkeıt, Köln 1987, S. 161 .

S A.J. Wicund, 3. 2. O., S.171. 
men, existicren Erschwernisse für Ausländer beim Zugang zu einzelnen Medienberulen. In Großbritannien gibr es seit 1981 einen »Broadcasting Acr", der vorschreibr, daß sproper proportions of programm material of British origin and of British performance" stammen sollen. Ausgenommen Sendungen von kulturellem, archivarischem oder bildungspolitischem Wert soll im Privaternsehen 86 Prozent der Sendezeit auf britische Programme entfallen. Auch in Belgien, den Niederlanden und den skandinavischen Ländern existieren vergleichbare Quorenregelungen.

Im Frühjahr 1989 haben die Mitgliedstaaten des Europarats eine Konvention über grenzüberschrcitendes Fernsehen ${ }^{6}$ angenommen, die EG hat ein Jahr zuvor eine Richrlinie ${ }^{7}$ zu diesem Problem erlassen. Die EG-Richtlinie verfolgt das Ziel, vor 1993 den 12 Mirgliedscaaten einen umfassenden Binnenmarkt für Rundfunkprogramme zu ermöglichen. Die in den Mitgliedstaaten ausgestrahlten Fernsehprogramme sollen in der gesamten Europäischen Gemeinschaft unbehindert verbreitet und emplangen werden können. Zur Untcrstüızung des europäischen Fernsehens heißt es in Art. ${ }_{4}$ der EG-Richtlinie: „Die Mitgliedstaaten achren im Rahmen des praktisch Durchfülırbaren und damir angemessenen Mirceln darauf, daß die Fernsehveranstalter den Hauptanteil ihrer Sendezeit, die nichr aus Nachrichten, Sporiberichicn, Spielshorws oder Werbe- und Telercxtlcistungen besteht, der Sendung von europäischen Werken im Sinne des Artikel 6 vorbehalten ist ..... Während sodann die EG-Richtlinie dicse Bestimmung dahingehend konkretisiert, daß $\cong$ Fernsehveranstalter mindestens $10 \mathrm{v}$. Fl. ihrer Sendezeit ... der Sendung europäischer Werke von Herstellern vorbehalten, die von den Fernsehveranstaltern unabhängig sind $\alpha$, enthält die Europarat-Konvention kcinc entsprechende Regelung.

Kulturpolisch von zentraler Bedeutung sind die Vorschriften, die die Länge der Werbezeit betreffen. In der Regel darf der Anteil für Werbung nicht mehr als is Prozent der täglichen Sendczeit betragen. Wichtiger als die Länge ist jedoch die Lage der Werbezeit. Denn sie entscheidet darüber, ob kulturell anspruchsvolle Werke ohne Unterbrechung durch Werbung aufgeführt werden dürfen. Arc. 10 der EG-Richtlinie sieht vor, daß dic "Sendezeit für Werbespors innerhalb einer Stunde ... 20 v.H. nicht überschreitena darf. Wer sich angesichrs dieser Regelung an amerikanische Verhältnissc exinnert sicht, wird von Art. 1 i scheinbar wieder beruhigt, indem dieser für die Fernsehwerbung statuiert, daß sie zwischen den Sendungen eingefügt werden muß. Dic Ausnahmen von dieser Vorschrifr freilich lassen dann doch zu, daß Kino- und Fernsehfilme nach jeweils 45 Minuten unterbrochen werden können. Ist cin Film längcr als 90 Minuten, dann dürfen bereits nach weiteren 20 Minuten Werbespors gesendet werden. Für allc anderen Sendungen sind die Regelungen großzügiger ausgestalrat. Hier gitt der Grundsatz, daß zwischen zwei aufeinanderfolgenden Unterbrechungen cin Abstand von mindestens 20 Minuten liegen muß. Auch politische Magazine, Dokumentarfilme, religiöse Sendungen und Kinderprogramme sind für Werbespors zugclassen. Nur dann, wenn die Sendezeit weniger als 30 Minuren beträgt, ist keine Werbung erlaubr. Für deursche Verhältnisse bedeuret dies, daß die EG-Richtlinie die Ausstrahlung von Werbespors in einem Umfang gestattet, wie es bisher nicht möglich war. Was bereits in vielen Ländern prakciziert wird, Kunstwerkc selbst als Medium für Werbung zu benuızen, kann nun auch in der Bundesrepublik geschehen. (Die EG-Richtlinie enthäls des weiteren Vorschriften für Werbung für alkoholfreie Getränke, Sponsorship, das Rechx auf Gegendarstellung und den Schutz der Minderjährigen.)

6 Siche I. E. Schwanz, Fernsehen ohne Grenzen: Zur Effekeıvität und zum Verhillenis von EG-Richdinte und Europarats-Konvention. Europarecht Heft 1/1989, S. 2, FN 2.

7 Richelinic des Rates zur Koordinienung von Reches- und Verouliungsvorschrifien über die Ausubung der Rundfunkfreihcil, ABI. Nr. C 110 vom 27.4.1988, S.3 
Während die Vorschriften der EG-Richtlinie und der Europarat-Konvention, die sich auf die Werbezeit beziehen, weitgehend anerkannt sind, ist um die Quorenregelung ein heftiger Streit entbrannt, obwohl weder die Richtlinie noch die Konvention cinen verpflichrenden Charakter für den nationalen Gesetzgeber haben. Gegen Quocierungen im Medienbereich ist eingewender vorden, sie würden den ungehemmten Informationsfluß zwischen einzelnen Ländern - Ziel einer europäischen Integration auch auf kulurellem Gebiet - beeinträchtigen. Quocierungen im europäischen Medienbereicl) seien ein Widerspruch in sich. Sie intensivierten staacliche, bürokratischc Eingriffe in dic Kultur statt die für kulturelle Prozesse erforderlichc freie Kommunikation zu unterstützen.

Von Seiten der Politik ist es besonders der baden-württembergische Ministerpräsident Lothar Späth, der sich gegen den »kulturellen Protektionismus» ausspricht. Er sieht in der "kulturellen Abschottung* Europas von den Vereinigten Staaten von Amerika einen weklatanten Eingriff in die Rundfunkfreiheit der Länder und einen Verstoß gegen Grundbedingungen des offenen kulturellen Zusammenlebens. $\pi^{y}$ Er tritc zwar auch, wie die Befürworter der Quorierung, dafür ein, die Konkurrenzlähigkeit curopäischer Film- und Fernsehprodukte gegenüber denen der USA zu verstärken, will dies aber nicht im Wege administratjver Strategien erreichen. Er sieht im Aufbau eines europäischen Kulturkanals, der aus dem keimförmig bereits existierenden deutsch-französischen Kulturkanal hervorgehen könntc, eine Möglichkeic der unbürokratischen Europäisierung des Fernsehens. ${ }^{9}$ Freilich sind die Gefahren dieser Konzeption unüberschbar. Wie die Privatisierung öffentlich-rechtlicher Medien, wie Rundfunk und Fernsehen, zu cinem in der deurschen Mediengeschichte beispiellosen Qualitätsverlust geführt hat, drohr auch ein von Konzernen wie Berlusconi, Maxwell, Bertelsmann oder Großverlagen wie Burda, Bauer und Springer rvesentlich bescimmter europäischer Kulturkanal mehr wirtschaftlichen Interessen zu entsprcchen, als die Herausbildung eincr eigenständigen europäischen Kultur zu unterstürzen. Derzeit besteht die Alternative zwischen einer bürokraisch, von Brüssel aus verordnecen und einer von Konzernen bestimmten europäischen Medicnkultur, dic in beiden Fällen Kultur fortschreitend zu nivellieren droht. ${ }^{10}$ Das eingangs skizzierte Dilemma einer Verrechelichung von Kulcur, gar auf europäischer Ebenc, kommc in der gegenwärtigen Medienpolitik auf den Begriff: Ohne Verrechtlichung (durch Anti-Trust-Regelungen) drohen die Massenmedien zum Spielball ökonomisch mächriger Konzerne zu werden, ein verrechtlichtes Mcdienwesen hingegen droht seine nationalc Eigenart unter dem Eingriff staatlicher Institurionen zu verlieren.

Die Kritik an Quocierungsmodellen ist der Grund dafür gewesen, daß in allen europäischen Ländern pauschalc Regelungen hinter differenzierten Quoticrungen zurückgetreten sind. Diese sehen für einzelne Programmsparten besondere Regelungen vor, enthalten Vorschriften für einzelne Berufsgruppen oder für spezifische Sendezeicen. Pauschalc Quocierungen, dic generelle Begrenzungen der Auslandsanreile im Gesamıprogramm vorsehen, gibe es beispielsweise in Italien. Dorr sind die Quoten im privaten Fcrnsehen ab ig 96 auf 40 Prozent Inlandsanteil der Produktion fcstgeseczt worden. Das nordrhein-westfälische Rundfunkgesecz von 1987 sieht eiaen entsprechenden Anteil von so Prozent vor. Differenzierte Quotierungen gibt es in den unterschiedlichsten Varianten. Begrenzungen der Auslandsanteile für die Haupsendezeiten zwischen ig und 23 Uhr sehen Regelungen in Großbritannien

8 1992. Der Traum von Europ3, Stutgarn 1989. 5. 227.

9 A.1. O., S. 240 .

10 St. Auseen/H. Cornel, Vorwort. Kultur-Markı Europa, in: Internationale Culturele Stıcheng/Kulcurpo-

litusche Gescllschafe (Hrsg), Kultur-Markt Europa, Köln 1989, S. 10. 
und Kanada vor. In Frankreich und Italien sind Quotierungen nach einzelnen Programmsparten (wic Musik, Spielfilme, Dokumentarsendungen, Kinderfilme) ausgeprägr anzutreffen. Neben pauschalen und differenzicrten Quotierungen lassen sich noch positive Quotierungen unterscheiden. Diese legen bestimmte Mindestsendezeiten oder -produktionen für einzelne Programmformen fest. In den Niederlanden etwa hat der Geserzgeber 20 Prozent der Sendezeit für einheimische Kulturangebote vorgeschen, in Italien müssen so Prozent der Produktionen einheimisch sein."

Einer Harmonisienung « dcr Programmpolisik öffentlich-rechtlicher Medien stchen die Besonderheit der nationalen Medienpolitik gegenüber. Je stärker sich die Tendenz durchsetzt, differenziertc Quorierungen cinzuführen, desto mehr klaffcn die Regelungen einzelner Länder auseinander. Hier worden die Grenzen einer Europäisicrung kultureller Institutionen sichtbar. Mehr noch als für den Austausch standardisicrter Güter gilt für kulturclle Produkıe, daß die je spezíische nationale Besonderheir einer internarionalen Vereinheirlichung Schranken serzt, die zu überschreizen nur mit hohen sozialen Kosten möglich ist. Es ist nicht nur der Empfchlungscharakter kulturrechtlicher Vorschriften, der ihre praktssche Bedeutung für die einzelnen Länder relativiert, sondern auch die Eigenart nationaler kultureller Systeme. So muß man beispiclsweisc die Regelungen der EG-Richrlinie, die Umfang und Lage von Werbezeicen in Rundfunk- und Fernsehprogrammen normieren, vor dem Hintergurnd der kuluurpolitischen Praxis in der Bundesrepublik berrachien. Da zumindest in den öffentlich-rechdichen Medien seit vielen Jahren eine bestimmte Praxis im Umgang mit Werbesendungen besteht, wird diesc durch europareclitlichc Vorschriften nicht verändert wcrden. Viclmehr dürfte es urngekehrt sein: Der in den privaten Medien verstärkte Einsalz von Werbung auch im Verlauf von Sendungen kann sich auf europaweite Normicrung berufen, um den nationalen Gesetzgeber zum Handeln zu veranlassen.

Neben den Problemen der Umsctzung der EG-Richtlinie in nationales Recht bestehen auf europäischer Ebene cine Reihe rechtlicher Unklarheicen. Während auf der einen Scice der Rat im Wege der EG-Richtlinic eine ungehinderte Fernsehdistriburion in den 12 Mitgliedstaaten ermöglichen wolltc, setzten sich dic Gegner des Binnenmarkses für cine "Europäische Konvention über das grenzüberschreicende Fernsehen ein. Die von 22 curopäischen Staaren am I J.3. 1989 vereinbarte Konvention, cinc Institution des Völkerrechts, unterscheidet sich in Umsctzung, Reichweite und Geltung von der EG-Richtlinie. Bei der Richelinie sind alle Mitgliedstaaten zur Umsetzung verpflichtet, bei der Konvention nicht. Die Umsetzung der Richrlinic hat in einer bestimmten Zcit zu erfolgen, die der Konvention nicht. Im Rahmen der Konvention können einzelne Mitgliedstaaten bestimmte Vorschriften für sich ausnchmen, die Richtlinie ist in toto verpflichtend. Entsprechend kann die Konvention von einzelnen Staaten gekündigt werden, dic Richtlinie nicht. Und schließlich wird die Beachrung der Richtlinic durch Behörden der Mitgliedstaaten überwacht, die wiederum von einer rechrlich kontrollierbaren Institution, der Kommission, übcrprüft wird. Ein entsprechender Rechrsschutz bestcht für die Kontrolle der Einhaltung der Konvention nicht. Deshalb gewährleister der Europäische Gerichtshof nur die cinheitliche Auslegung der Richrlinie durch die Gerichte der Mitgliedstaaten. Die Auslegung der Konvention hingegen ist altein Angelegenheit der nationalen Gerichte. Der Medienrechter Schwartz kommt deshalb zu der Einschätzung, daß ^die Geltung des Übereinkommens zwischen den Mitgliedstaaten der Gemeinschaft ... die heute bestchende Lage hinsichtlich der Kontrolle von

1) Eine detaillieret Auslistung findet sich bes A.J. Wiesand, a. a. O. 5. 1861. 
grenzüberschreitenden Programmen sowic der Rundfunkwerbung sogar verschlechtern* würde. Denn es bestehen national vielfach liberalere Regeln. Damit würde die Errichtung des Binnenmarkes niche erlcichtert, sondern erschwert. " ${ }^{12} \mathrm{Im}$ Bereich des Femsehen bestchen nach der gegenwärrigen Rechtslage zwei europäische Recheszonen: eine der Gemeinschaft und eine der übrigen demokratischen europäischen Staaten.

\section{Institutionen europäischer Kulturpolitik}

Imponierend ist die Bilanz eines curopäischen Kulurverwaltungsrechts bislang nicht. Niche überraschend ist deshalb die Klage eines seit vielen Jahren auf diesem Gebict Engagierten: „Die Kulturpolitik der Europäischen Gemeinschaft (EG) kommt nicht voran. Das betrifft nicht nur ihre nach wie vor äußerst bescheidene finanzielle Ausstattung ... sondern betrifft vor allem die mangelnde rechtliche Absicherung dieses Politik-Feldes." 'S Zwar ist die Zahl der mit Kultur befaßten Instutionen und Vereinbarungen auf europäischer Ebene groß, ihre tarsächliche Bedeutung hingegen nachrangig.

Das Internatzonale Erziebungsbüro in Genf isı die älıcste internationale Organisarion im Bereich der Kultur, der seir ihrer Gründung 1929 rund 90 Mirgliedstaaten beigerreten sind. Der Einfluß auf das curopäische Kulturverwaltungsrecht ist deshalb gering, weil das Internationale Erzichungsbüro neben einer jälırlich in Zusammenarbeit mit der UNESCO veranstalteten Internationalen Erzichungskonferenz keine weiterreichenden Aktivitäten entfaltet. Die 1961 mit wirtschaftspolitischer Ziclsec\%ung gegründete Organisation für wirtschaftliche Zusammenarbeic (OECD) hat in den vergangenen Jalıren zwar auch Fragestellungen aus dem Bereich wissenschaftlich-technologischer Enrwicklung aufgenommen. Über den Erfahrungsaustausch zowischen den Mitgliedstaaten gehen diese Koopcrationen aber nicht hinaus. Weitergehend sind dagcgen Kulcurinstitutionen, die im Rahmen der Europäischen Gemeinschaft entstanden sind. Im Montanbereich fördert dic Hohe Behörde nach Art. $55 \$ \mathrm{EGKSV}^{24}$ technische und wirtschaftliche Forschungen und ist an der Berufsschulung beteiligt (Art. 56). Zudem legen die Mitglicdstaaten gemeinsam berulsrechtliche Begriffsbestimmungen bei der Herstellung der Freizügigkeit der Kohie- und Stahlfacharbeiter fesi (Arr. 69). Im Rahmen der Europäischen Wirtschaftsgemeinschaft koopericren die Mitglicdstazten im Bereich der Universitäten und der Berufsschulen. Richtlinien für die gegenseitige Ancrkennung von Diplomen, Prüfungszeugnissen und anderen Qualifikationen hat die Gemeinschaft nach Art. 57 Abs. I EWGV's cbenso crlassen wie zur Koordinierung der Reches- und Verwaltungsvorschriften über die Aufnahme und Ausübung selbständiger Tätigkeiten. Der Euratom-Vertrag schlicßlich enthält Normen zur Förderung der Forschung im Bereich der Kernenergie (Art. ${ }_{4} \mathrm{ff}$. EAG-Vertrag), zur Verbreitung wissenschaftlicher Erkennenissc (Art. เ2 ff.) und zum Gesundheitsschutz (Art. 30 If.)..$^{16}$

12 I. E. Schwartz, 2.2 O., S.8.

13 O. Schwencke, Europa ist mehr als die EG - Kuleurpolitik in der Gemeinschaft, Kulcurpolitusche Mitteilungen $\mathrm{Nr}, 4$, 11/1989, 5. 18 ff

I4 Venmg iiber die Grundung der Furopaisclien Gemeinschafi fur Kohle und Suahl vom 18.4.1951, BGBI. 1952 1I, S. 447.

is Verirag xur Gründung der Europäischen Wirtsehafesgemeinsehafe vom 25. 3. 1957, BGBI. II, S. 766.

16 Vertrag zur Gründung der Europäischen Aromgemenschaft vom 25. y. 1917. BGBI. II, S. 101.1. 
Über diese, unmittelbar auf den wirtschaftlichen Zweck der jeweiligen Vereinigung gerichteten Organisationen hinaus, sind kuluurelle Institutionen gegründet worden, die in ihrer Wirkungsweise weitgehend unabhängig von ökonomischen Oprionen sind. Dazu gehörcn die Europäischen Schulen, die von einer selbständigen internationalen Institution finanzierr und̀ verwaltet wenden und in denen nach eigenständigen, international vereinbarten Lehrplänen unrerrichtet wird. Das im Verglcich zu anderen curopäischen Projekten relativ reibungslose Zustandekonmen der Europäischen Schulen dürfte in der prakeischen Norwendigkeit begründet gewesen sein, die Kinder der Beschäftigten von europäischen Institutionen qualifiziert zu unterrichten. Auch das Europäische Hochschulinstitut in Florenz, wiewohl nicht zur Qualifizierung von Bediensteten der Gemeinschaft gegründet, gehört in den Kontext relativ eigenständiger kultureller Inscitucionen.

Regetren die dargestellten Verträge kulturelle Fragen im Kontexr ökonomischer Zusammenhänge, so sind die multilateralen Abkommen, dic im folgenden dargestellt werden, primär kulturpolitische Vereinbarungen. An erstcr Stelle ist der Europarat (Straßburg) zu nennen, zu dessen Aufgaben unter anderem der Abschluß von Abkommen und ein gemeinschaftliches Vorgehen auf kulturellem und wissenschaftlichem Gebiet gehört. Von kultureller Bedeurung ist der in Art. (b der Satzung des Europarats ${ }^{17}$ erwähnte Schutz der Menschenrechte und Grundfreiheien sowic ihre Forentwicklung, der unterstiizzenden normariven Ausdruck in einer Konventian zum Schutz der Menschenrechte und Grundfreibetlen vom 4.11. $1950^{18}$ gefunden hat, dic dic Mitglicder des Europarats vercinbart haben.

Historisch lassen sich zwei Phasen kultureller Aktivitäten des Europarats unterscheiden. Da ist zunächst die Zeit von der Gründung 1949 bis zur Errichtung des Rats für kuhturelle Zusammenarbeit (1962). In dieser Phase schlossen dic Mirglicdstaaten des Europarats eine Reihe von Abkommen, die vorwiegend die Angleichung formaler Qualifikationen in den cinzelnen Ländern zum Ziel hatten (z. B. Europäische Konvention über die Glcichrvercigkeit von Reifezeugnissen vom 11. 12. 1953; Europäisches Übcreinkommen über dic Gleichwertigkeit der Studienzeiten an den Universitäten vom is. 12. 1956; Europäisches Abkommen über die Anerkennung von akademischen Graden und Hochschuizeugnissen vom I4. 12. 1959). Darüber hinaus vercinbarten sie das Europäische Kuhurabkommen (vom 19.12.1954), das in der Zwischenzeir mehrere Male revidiert worden ist. Oppermann schärzt die praktische Relevanz der Abkommen der ersten Phase kultureller Tätigkeit des Europarats gering ein, weil die vercinbarten Verpflichsungen bcschränkt sind und durch Ausnahmen wieder relaciviert werden. Hinsichrlich des freien Transfers von formalen Qualifikacionen sei die Konvention über die Gleichwertigkeit von Reifezeugnissen am folgenreichsten gewesen, die aber gerade deshalb in der deutschen Kulturverwaltung umstritten ist. ${ }^{19}$

Anfang der secliziger Jahre wurde dic kulturelle Arbeir des Europarats reorganisiert und intensiviert. Organisatorischcr Ausdruck dessen war die 1962 erfolgte Gründung des Rats für kulturelle Zusammenarbett (CDCC), mit dessen Tärigkeir die kulturellen Akrivitäten des Europarats Mitte der sechziger Jahrc 42 Prozent der Gesamträtigkeit ausmachten. Die Arbeir des Rats für kulturelle Zusammenarbeit, dem neben den 17 Mightiedstaaten des Europarats Jugoslawien und der Heilige Stuhl (also die MLitglieder des Europäischen Kulturabkommens) angehören, wird von drei Ausschüssen bestimmt: dem für akademische Lehre und Forschung, für das Schul- 
und Fachhochschulwesen und für außerschulische Bildung und Erzichung. Die Bundesrepublik wird im Rat für kulturclle Zusammenarbeit durch das Auswärige Amt und die Kultusministerkonferenz repräsentiert. In den ständigen Ausschüssen sind Vertreter der zuständigen Behörden von Bund und Ländern tärig. Die Aufgaben des Rats bestehen vorwiegend darin, nationale Projekte aus den Bereichen der ständigen Ausschüsse zu vergleichen und Koordination oder Kooperation herzuscellen. Daneben soll der Rat in den fünfziger Jahren begonnene Projekte, wie Stipendien- und Austauschprogramme, Förderung von Publikationen und Ausstellungen, Verleihung von Filmpreisen, Veranstaltung von Seminaren usw. fortführen. Auch hinsichtlich der Tätigkeit des Rats für kulturelle Zusammenarbeit gelangt Oppermann zu einer vorsichrig pessimistischen Einschätzung. „Die Tätigkeit des Rats für kulturelle Zusammenarbeit bewegt sich im Rahmen begrußenswerten, aber wcitgehend unverbindlichen geiscigen Austausches sowic der finanziellen Förderung gervisser Vorhaben mit relativ beschránkten Mitteln. $\alpha^{20}$

In den vergangenen Jahren indes hat sich ein Bedeutungswandel der Kultur vollzogen, der auch Europa erfaßt hat. 1983 beschlossen dic im Europäischen Rat zusammengeschlossenen Staats- und Regierungscheís eine sengere kulcurelle $\mathrm{Zu}$ sammenarbeit, um das Bewußtsein eines gemeinsamen kulturellen Erbes als Teil der curopäischen Identiät zu lestigen . Zwei Jahre später wurde eine jährlich wechsclnde *Kulturhauptstadt Europa cingerichtec, nachdem zuvor bereits ein $n$ Europäisches Jahr der Denkmalspflege» ausgerufen worden war. Diesen Iniciativen folgeen curopäische Jahre der Musik, des Films und des Fernsehens. Den vorläufigen Schlußpunkr einer Reihe weiterer Inititativen" bildet cine Entschließung des Europäischen Parlaments vom 17.2.1989, die sich auf cinen Rahmenplan der EGKommission über sImpulse der gemeinwirtschaftlichen Aktion im kulturellen Bereicly bezieht und diese konkretisiert. ${ }^{22}$

Entkleidet man die Entschließungssprache von Parlamentariern ihrer hölzernen Form, dann bestehe der Grundgedanke der "Entschließung « darin, daß ein ökonomischer Zusammenschluß der europäischen Länder nicht vorstellbar ist, wenn er nicht von kulturellen Kooperationen vorbercitec und begleiter wird. In der modernen Managementsoziologic gilı der Gedanke, daß die Effekrivität ökonomischen Handelns auch von außerökonomischen Kriterien, wie kulturellen, abhängt, keinesrvegs mehr als originell. In dem Maße, in dem die Überlegung an Plausibilität gewonnen hat, daß die Ware Arbeitskraft ein komplexes Gut ist, das sich nicht auf ökonomische Kalküle reduziercn läßt, begann in der Soziologic cine Wiederentdekkung kultureller Bedürfnisse als Element der menschlichen Arbeitskraft. ${ }^{23}$ Wenn das Europaparlament der Auffassung ist, obne kulturelle Kooperation sei kcine Wirtschaftsintegration vorstellbar, dann folgr es diesem Grundgedanken der modernen Industriesoziologie.

Die Renaissance des Subjektbegriffs in der Industriesoziologie ist aus empirischen Beobachcungen über Veränderungen in modernen Produkcionsprozessen und im Verhälenis von Arbeitern und Management hervorgegangen. Auf der Ebene der Europapolitik wird die Notwendigkeit, kulturclle Bedürfnisse zu berücksichtigen,

20 Th. Oppermann, a.2. O., S.621. Zu welteren turopäischen Kulturprojekien wic der Konferenz der Europaischen Erzsehungsminisier, dem Europäischen Schuleag und dem Europa-Kolleg Brügge vgl. Oppemann, a.a.O., S.625.

a E. Krıngs, Kulturpolitik ım "Reıch der Zwölf*, in: Incernäıonale Culıurcle Seıcheıng/Kulnopolieısche Gescllschaft (Hrsg.), 1. a. O., S. 94

12 Abgedruckt in: Kulturpolicusche Mitreilungen Nr. 45 I1/rg8g, S. igft.

23 Exemplarisci) datür ist dic Debatte um das Buch von H. Kern/M. Schumann, Ende der Arbeitsteilung? Ralionalisıerung in der industriellen Produktion, Munchen ig $S_{4}$. 
nicht aus Veränderungen im Verhältnis von Kapiral und Arbcit begründet. Hier geht cs vorwiegend um Bcziehungen zwischen Kapital und Kapital oder Arbeit und Arbeit. Aber auch diese Beziehungen werden nur dann einen wirtschaftlichen Integrationsprozeß vollziehen können, wenn dieser von kulturellen Anglcichungen begleitet ist. Diese ldee ist angesprochen, wenn es in der Entschließung heißt, daß der wgroße Binnenmarkt keine wirklichen Fortschritre in Richtung auf die ... Europäische Union bedeuten... (wird), wenn er sich nicht $/ u$ eincm Sozialraum entwickelt, in dem die Erfindungen, die Forschung, das Schulwesen, die Berufsausbildung, das Kunstschaffen, dic Neubelebung und Erneuerung des kulturellen Erbes und die Kulturindustrien ebenfalls Zeichen des Foreschritts des europäischen Aufbauwerks sind, es bcgleiten und ihm sogar den Weg bereiten. "24 $^{24}$

Kultur nun versecht das Europaparlament in einem umfassenden Sinn. Es sind nicht nur die Ausdrucksweisen, vermittels derer Personen, soziale Gruppen oder nationale Bevölkerungen die Komplexität ilhrer Probleme verarbeiten, die das Parlament unter Kultur versteht. Kultur soll mehr sein als künstlerische Darstellungsformen. Wenn das Europaparlament darauf hinweist, daß die Vielscitigkeit der Kulkur verlange, $*$ daß das Forbestehen gesellschaaftlicher Randgruppen ..., die vom Bildungswesen nicht erfaßt werden, dic keine Berufsausbildung crhalten und keine Aussicht auf eine Verbesserung ihrer Lage haben, nicht lingenommen wird ${ }^{2}$ " dann verstehc es unter Kultur auch cin Engagement für soziale Probleme. Wenn ich die Entschließung richtig interpretiere, dann soll Kultur wenigstens in dreifacher Weise von Bedeutung sein: Sic soll erstens den ökononischen Integrationsprozeß unterstützen und damit ermöglichen, zweitens den unterschicdlichen lokalen, regionaien und nationalen Gruppen Ausdrucksmöglichkeiten und Anregungen vermitteln und drittens einen politischen Beitrag zur Lösung drängender sozialer Probleme leisten.

Was das konkret bedeuten soll, legt das europäische Parlament in 68 Empfehlungen dar, die die Komplexe "Bildungswesen «, "Sprachen «, "gemeinschaftliche kulcurelle Dimension “, "audio-visueller Bereich “, "Kenntnis des kulturellen Europas $\alpha$, "Vercinigungen und Stiffungen $\alpha$ und "Dialog zwischen den Kulturen $*$ umfassen. Hier finden sich Wünsche nach einer besseren scatistischen Kennunis des kulturellen Niveaus von Europa, Beseitigung des Analphabetentums sowic Unterstürzung der kulturellen Wetrbewcrbsfähigkeit und der »anderen "Kultur. Aber auch detaillierte Überlegungen zur Mehrwertsteuer bei Büchern und Zeitschriften, der Verringcrung von Abgaben auf Schallplatten, Phonogramme oder Videogramme im Rahmen eines curopäischen Klassifizierungssystems, der Übersetzung von bedeutenden Werken der europäischen Kultur, der Harmonisierung der Normen für die Kacalogisierung in Bibliorheken, der Einführung moderner Darcnverarbeitung im Bibliothekswesen, der Zusammenarbeit von kulturellen Stifungen werden dort angestellt. Schließlich soll eine europäische Kulturpolitik auch einen Beitrag zur Auflösung des Ost-WestGegensatzes leisten.

Analysen, die sich nit der Wirkungsweise kulturverwaltungsrechelicher Vorschrilten in Europa beschäftigen, betonen mehr Grenzen und Restriktionen als Innovationen. Der Kultursoziologe Andreas Wicsand hat solche Grenzen anhand ciner empirischen Untersuchung dargetan, die die Probleme des grenzüberschreitenden Verkehrs mit Werken der Bildenden Kunst zum Gegenstand hatre. Wälirend auf der einen Seitc in den vergangenen Jahren der internationale Kunschandel (vorwiegend für Ausstellungen) zugenommen hat, vergrößerten sich die beim Grenzübertritt 
auftretenden Probleme, so daß mit ihren Werken reisende Künstler häufig lange

Wartezeiten an den Grenzen verbringen mußten. Die Möglichkeiten für Probleme sind vielfältig: für die Mehrwert- bzw. Einfuhrumsatzsteucr müssen Künstler an der Grenzc eine Kaution hinterlcgen; einige Länder verlangen die entgeleliche Einschaltung eines Zoll- oder Transitagenten; Bescimmungen des „Kulturgutschutzes», zum Schutz wertvoller Sammlungsstücke gedacht, erschweren auch den Handel mit anderen Kunstwerken; die Fracht- und Postgebühren sind ungleich höher als dic für andere kulturelle Waren, wie ecwa Bücher. Diese vielfältigen zeitlichen und finanziellen Belastungen haiten viele Künstler davon ab, am internationalen Kunstmarkc zu parsizipieren. Weil die administrativen Schwicrigkeiten so umfangreich sind, bedienen sich einflußreiche Künstler oder Institutionen diplomatischer Kanäle, um auf dicse Weise einen reibungsloseren Austausch zu erreichen. Das cinschlägige -Florenz-Abkommen*, das den Handein mir Kunsıwerken erlcichrern soll, spict nach Aussage der Befragren in der Praxis keine nennenswerce Rollc. nDer grenzüberschreitende Vcrsand von Kunstwerken «, resümicre Wiesand, sist teuer, komplizicr und vielfach cingeschränkt.a. ${ }^{26}$

Der Problematik eines geringen nacionalen Einflusses curopäischer kulturverwaltungsrechılicher Vorschriften unterliegen alle Vorschläge, die gegenwärtig für eine kulturpolitische Ergänzung des 1992 in Kraft cretenden europäischen Binnenmarktcs gemacht werden. Diese Vorschläge, am weitcstgehenden von der „Gruppe der SPD-Abgeordneten in der Sozialistischen Fraktion des Europäischen Parlaments* formuliert, lassen sich nach folgenden Sachgebicten strukturieren:

- Haushaltspolicik (cin Prozent des Haushalts für kulturelle Ausgaben),

- Sozialpolitik (Zahlung des Arbeitgeberanteils zur Sozialversicherung aus einern von Verłagen und dem Kunsthandel finanzierren Fonds, damic Künstler der Sozialversicherung beitreten können);

- Sreuerpolitik (Abbau der Doppelbestcucrung von Künstlern im Hcimat- und Gastland; Übertragung der Steverharmonisierung vom ökonomischen auf den kulturellen Bereich unter Beibehaltung des errmäßigten Steuersatzes für kulturelle Leistungen; insbesondere Abbau der unterschiedlichen sceuerlichen Behandlung von Büchern und anderen Princmedien);

- Arbeirsmarkcpolitik (Beschäfrigungsinitiativen für "Kulturschaffendle«; Aufau einer wetrbewerbsfähigen europäischen Kulturinduscrie);

- Urheberrechr (Schutz von Autoren- und Verlegerrechten, die durch Forokopicren und Raubdrucke verletzt werden; Vergütung für Zwcit- und Drittauswertung);

- Bibliorhekswesen (Einführung ciner curopaweiten Datenyerarbeitung, die einen raschen Zugriff auf alle Werke in europäischen Bibliocheken gestantet; Errichtung einer Europäischen Bibliorhek);

- Zollwesen (Entbürokracisierung von Zollvorschriften, die den Austausch und auf, bzw. Verkauf von Kunstwerken betreffen);

- Medienpolitik (Europäische Rundfunkordnung, die den freien Informationsiluß über die nationalen Grenzen hinaus verbesscrt; Maßnahmen zur Verhinderung von Meinungsmonopolen und Machtkonzentrationen im Medienbereich; Vereinbarung ciner europäischen Medienkonvention, die folgendes enchält: Recht auf Gegendarstellung, Jugendschutz, Werbebegrenzungen, Urhebcrrecht, Quoticrung zur Garancic eines Mindestanceils europäischer Produktionen; europäische Koproduktionen, Aufbau transnationaler Programmaktivitäten; Verbesserung der internationalen Wettberverbsfähigkeit europäischer Programme);

26 A. 1. O., S.137. 
- Bildungspolitik (Verbesserung von Sprachkenntnissen; Vergabe von Stipendien an Schauspieler, Regisseure und Schrif(steller);

- Denkmalspflege (Schutz von Baudenkmälern vor ökologischen Einwirkungen);

- Forschungspolitik (Errichtung eines Institurs für Osteuropa-Studien)."

Es sind freilich nicht nur beschränkte Einwírkungsmöglichkeiten von Institurionen auf europäischer Ebene und mangelhafte normative Regelungen, dic die geringe praktische Relevanz eines europäischen Kulturverwalcungsreches begründen. Es ist auch dic Konstruktion eines kulturellen Europas, die eine Fülle von Problemen hervorbringt, dessen Ausdruck die geringe Relevanz des curopäischen Kulturverwaltungsrechts ist.

\section{Bedingungen eines europäischen Kulturverwaltungsrechts}

Gleichviel wclches Rechtsgebict man analysiert, wird es auf die Ebene der Europäischen Gemeinschaft transportiert, dann sind Klagen über die mangelnde Integrationsfähigkeit mic Rechtssystemen der anderen Länder so unüberhörbar, daß sie die Hoffnungsschimmer auf ein gemcinsames Europa überschatten. Die geringsten Fortschritte hin zu einem europäischen Recht indes scheint bislang das Kulturverwaltungsrecht gemacht zu haben. Allc Europaexperten sind sich eirig, daß neben den seit den fünfziger Jahren existierenden Initiativen für gemeinsame, rechtlich abgesicherte kulcurelle Institutionen in den vergangenen Jahren, in denen Europa zum großen öffentlichen Thema geworden ist, keine qualitativen Veränderungen stattianden. Der nachhinkende kulturverwaltungsrechtliche Integrationsprozeß ist fraglos eine Folge davon, daß das Projekt Europa ein wirschaftliches und kein kulturelles isc. Die Verbesserung nationaler Wetrbewerbsbedingungen durch einen intcgrierten europäischen Markt ist die Tricbkraft, die das Projekt Europa seit Beginn vorantreibr und zu cinem wirschaftlichen Erfolg zu bringen scheint. Kulturelle Integration war stess nur ein Appendix, dessen rudimentärcr Bedcutung nicht cinmal öffentlich widersprochen wurde. Das hat freilich den Vorzug, daß die beginnende Standardisienung des europäischen Mícdientvesens beeinflußbar blcibr. Denn was auf keinen Fall erfolgen sollte, ist eine Intcgration europäischer Kulturen im Sinne von Entdifferenzierung. Ganz im Gegenteil: Wenn hier von kultureller Integration die Rede ist, dann im Sinne einer verstärkten Differenzierung durch Kommunikation der europäischen Länder.

Zunchmend jedoch ist die Einsicht ins öffentliche Bewußtsein gedrungen, daß die Verbesserung des ökonomischen Warencausches nur dann zu errcichen ist, wenn auch Produzenten und Konsumenten der Waren in cinen kulturellen Dialog eintretcn. So enweiterte sich Europarecht als Wirtschaftsrecht bis zum europäischen Kulturverwaltungsrechr. Hier nun gehe es darum, sowohl der Europäisierung des kulturellen Warentauschs wie der Incernationalisierung der Arbeitskrafe dic normativen Bedingungen zur Verfügung zu stellen, die sie ermöglichen und sichern. Dies freilich ist nicht nur wegen der (noch) randständigen Bedeutung kultureller Integration so kompliziert, sondern vor allem weil kultureller Integration engere Grenzen geserz sind als wircschaftlicher. Der Austausch von Waren erfolge zwar durch das Handeln von Subjekten; diese treten jedoch allein in einen zweckrationalen Dialog, der mit Erreichen des Zwecks, dem vollendeten Warentausch, beendet ist. Ganz anders ist die kulturelle Interaktion strukturiert. Hier kann auch ein ökonomischer 
Zweck angestrebt werden (wie etwa die Verbesserung von Sprachkenntnissen zur Erhöhung beruflicher Qualifikation), dieser steht jedoch nicht im Zentrum der Interaktion. Das gemeinsame Lemen von Menschen, nicht der Austausch von Waren ist es, das dic Differenz bezeichnet. Und wo Subjekte in einen Dialog eincreten, bringen sie ihre je spezifischen kulturellen Tradicionen, der Sprache, der Religion, der Bildung und sozialer Verhalensweisen ein. Vermitzeln sich Waren, gleichviel aus welchem nationalen Kontext sie stammen, über ein abstraktcs Medium, das Geld, so Subjekte über soziale Interaktion.

Aus der sozialen Interaktion, die sich vermittels der Sprache und niche des Geldes entfaltet, resultieren nun die spezifischen Probleme einer Integracion kulturverwaltungsrechtlicher Bestimmungen. Die Probleme eines europäischen Kulturverwaltungsrechts werden durch die Schwicrigkeiten einer kulturellen Integration besrimme. Diesc sind offensichelich. Die Europawahl 1989, die cinen Ansieg rechtsradikaler Parteien verzeichnete, hat allc die auf das Problem kulturcller Integration verwiesen, die meinten, vom rehativ reibungslosen europäischen Warentausch auf ein holes Intcgracionsbedürfnis der Staatsbürger schlicßen zu können. In Wahlerfolg rechtsradikaler Parteien reflekticrt sich der Protest von Minderheiten darüber, daß die Subjekte das nachvollziehen sollen, was aus Gründen des Kapiralverwerrungsprozesses efforderlich ist. Soziale Voraussetzung für eine Integration verschiedener kultureller Systeme freilich ist, daß sic in ibrer Unterschiedlichkeit nicht nur akzepriert werden, sondern die Auscinandersetzung mit ihnen gerade wegen der Verschiedenartigkeir gesucht wird. Wenn von einer Integration kulcurverwaltungsrechtlicher Vorschriften die Rede ist, dann muß stets mitgedache werden, daß sie nur dann gelingen kann, wenn sic auf einer kulturellen Integration basicrt. Diese wiederum kann nicht bedeuten, daß die nationalen kulturellen Systeme zu einem neuen Multi-Kultursystem verschmelzen. Kulturelle Integration bedeuter vielmehr, daß Subjckte aus unterschiedlichen nationalen Zusammenhängen ihre je spczifischen kulturellen Eigenarten anerkennen und dennoch oder gerade deshalb in einen Dialog miteinander treten. Um es in der Terminologic zcitgenössischer Politiker zu formulieren: Eine multikulturelle Gesellschaft ist weder eine Idylle, in der sich unterschiedlichc Kulturen zu wechselseitigen Erfahrungen drängen (wie dies nicht selten im Umkreis der GRÜNEN gehoffe wird), noch ist sie cin Schreckbild, dem mit Anwendung disziplinarischer Strategien vorgebeugt werden kann (wie der Rechrsradikalismus suggeriert). Multikulturelle Gesellschaften sind vielmehr soziale Gebilde, die mit einem drastisch erhöhten Konflikıniveau konfrontiert sind, dem sic allein in der Weise adäquat entsprechen können, daß sie dieses akzcpticren und unrerschiedliche Formen der Konfliktbearbeitung und -lösung herausbilden. Die Tolcranz von kulcurcller Pluralität ist eine Bedingung multikultureller Gesellschaften.

Die normative Integration kulturell unterschiedlicher Sysreme ist aus diesen Gründen primär mit ihren Grenzen, nicht mit den Chancen für ein ncu entstehendos curopäisches Kultursystem konfrontiert. Dieser Ausgangspunkt ist konstitutiv für die Frage, wie die Entwicklungsperspektiven für cin europäisches Kulturverwaltungsrecht einzuschätzen sind. Dieses kann erfolgreich nicht mchr gesetzlich insritutionalisieren, als sozial erreichbar ist. Prinzipiell unproblematisch ist aus dieser Prespektive dic Harmonisierung gesetzlicher Regelungen, dic sich auf den Austausch von kulcurellen Gütern beziehen. Eine Entbürokratisierung von Zollvorschriften beim Kunsthandel, die Angleichung des Steuerrechts oder - um nur einige Beispiele zu nennen - Vereinheitlichungen im Bibliorheksrvesen sind deshalb denkbar, weil es sich in diesen Fällen um die Srandardisierung ökonomischer Abläufc, wenn auch auf dem Gebiet der Kultur handelt. Der Handel mit Kultur unterschei- 
det sich nicht prinzipieli vom Tausch jeder bcliebigen Ware. Integrationsversuche von Normen, die sich auf den Handel mit Kulcur beziehen, sind deshalb mit keinen größeren Schwierigkeıten belaster als die europäische Integration auf dem Gcbict der Ökonomic.

Die Grenzen eincr Veseinheitlichung normativer Regelungen im Bereich der Kultur werden sichtbarer, wenn man sich der Sozialpolitik zuwendet. Ohne in diesem Zusammenhang auf sozialrechtliche Details eingehen zu wollen, läßt sich doch das allgemeine Problem benennen. Die sozial- (wie auch die arbeits-) rechelichen Modelle der einzclnen europäischen Länder untcrscheiden sich in vielen Komplexen so grundlegend, daß der Versuch eincr Vereinheitlichung stets an die Schranken der diesen normativen Regelungen zugrundeliegenden unterschiedlichen sozialen Systeme stößr. So wird nur in solchen Ländern die Idce cines Fonds, aus dem der Arbeitgeberanteil zur Sozialversicherung für Künstler bezahlr wird, zu realisicren seın, die das zugrundeliegende Modcll ciner Koopcration von Arbeitgeber und Arbeitnehmer als gesellschaftpolitische Institution anerkennen. Dort hingegen, wo die Arbeitgeber-Arbcitnehmer-Bcziehungen als gegensätzliche anerkannt sind, wird ein solcher Vorschlag nicht oder nur eingeschränkt durchgesetzt werden könncn. Die Formen sozialer Institucionalisierung, die jeder normativen Regelung zugrunde liegen, so läßr sich die These resümieren, bilden eine Schranke für die Angleichung gesetzlicher Regelungen im Bereich der Kulrur.

Während dieser Typus von Schranke für cine Integration normativer Regelungen im sozzalen System der jeweiligen Gcsellschafe liegt, läßt sich eine weitere im kulturellen System der unterschiedlichen Länder lokalisieren. Im ersten Fall sind es je spezifische institutionelle Beziehungen, die einer Anglcichung entgegenwirken. Im anderen Falle sind es die tradierten Gewohnheiten der Subjekte, die einer Vereinheitlichung geseczlicher Regelungen zwar niche entgegenstehen müssen, diese aber doch erschweren können. Ein illustratives Beispiel dafür isı die EG-Richtlinie, die allgemeine Regelungen für Umfang und Lage von Werbung in Rundfunk und Fernschen enthält. Im Bereich der audio-visuellen Medien laben sich in den verschiedenen europäischen Ländern in hohem Maße unterschiedliche Formen im Umgang mit Werbung herausgebildet. Während etwa die bundesrepublikanischen öffentlich-rechtlichen Medien troč des verstärkten Einflusses der Werbung dieser einen randständigen Charakter gegenüber Programmen beimessen, ist dies in Ländern wie Frankreich oder Italien vollständig anders. Dort haben ökonomische Interessen viclfach eine solche Dominanz erlangt, daß cine von Wcrbung unberühre Aufführung von Kunstwerken nur selten möglich ist. Normacive Vereinheitlichungen haben in solchen Fällen, wenn sic überhaupt möglich sein sollten, allein den Charakter einer regressiven Nivellierung kulturcller Werke. Europäisierung wïrde dann bedcuten, daß dic je spezifische Eigcnart cines kulturellen Systems ausgelöscht wird.

Damit ist das Dilemma eines curopäischen Kulturverwaltungsrechts benannc. Es kann wirkungsvoll sein, sofern es sich auf die Berciche des kulturellen Warencauschs bezicht. Als Sozial-, Stever- oder Urheberrecht läßt sich eine Integration kulcurverwaltungsrechtlicher Vorschriften auf europäischer Ebene in der Weise vorstellen, wie es in Wirschaftsrecht üblich ist. Je mehr sich das Bemühen einer Vcrcinheitlichung kulturverwaltungsrechtlicher Vorschriften jedoch vom Warenaustausch entfernt und den kulturellen Ausdrucksformen von Subjekren näbcrt, desto geringer werden die Möglichkeiten für eine Harmonisierung. Es sind die Spezifika der unterschicdlichen kulturellen Systeme, die ihrer Integration Grenzen setzen. Anders formuliert: Ein curopäisches Kulturvcrwaltungsrcche wird sich dort herausbilden, wo es heure bereits als Praxis existiert, während es in den Bereichen scheitern wird, 
die sich wegen ihrer kulturellen Auronomic gegen Vereinheitlichung sperren. So geschen bestärigen die Versuche eincr Europäisierung rechtlicher Vorschriften die soziologische Erkenntnis, daß Recht nur dann institutionalisiert und implementien werden kann, wenn die sozialen Strukturen rudimentär herausgebiider sind, die es etablicren will. Und umgckehrt finder Recht dort seine Schranke, wo es etablierten sozialen Beziehungen widerspricht. 